\title{
Einsatz der Bone morphogenetic Proteins (BMPs) zur Behandlung von Pseudarthrosen - Effizienz und Therapieversagen
}

\author{
Use of Bone Morphogenetic Proteins (BMPs) for the Treatment \\ of Pseudarthroses - Efficiency and Therapy Failure
}

Autoren

Institut
M. Hausmann, S. Ehnert, V. Hofmann, S. Döbele, T. Freude, U. Stöckle, A. Nussler

BG Trauma Center, Eberhard-Karls-Universität Tübingen

Schlüsselwörter
BMP
Behandlung
Pseudarthrosen
TGF-beta
Smad
Key words
BMP
treatment
pseudarthroses
TGF-beta
Smad

Bibliografie

DOI http://dx.doi.org/

10.1055/s-0034-1368208

Z Orthop Unfall 2014; 152:

144-151 @ Georg Thieme

Verlag KG Stuttgart · New York . ISSN 1864-6697

Korrespondenzadresse Prof. Andreas Nussler

BG Trauma Center

Eberhard-Karls-Universität Tübingen

Schnarrenbergstraße 95 72076 Tübingen

Tel.: 07071/606-1065

Fax: 07071/606-1978

andreas.nuessler@gmail.com

\section{License terms}

\section{(ब) (1) $\Theta$}

\section{Zusammenfassung}

$\nabla$

In der Therapie von Pseudarthrosen der proximalen Tibia kommen seit einigen Jahren die humanen rekombinanten „bone morphogenetic proteins" (BMP-2 und BMP-7) zum Einsatz. Trotz limitiertem und spezifischem Einsatz als lokale Mediatoren der Knochenheilung können bisher keine Aussagen über den jeweiligen Erfolg der Therapie im Voraus getroffen werden. Die Regelmechanismen zeigen sich als wesentlich komplexer und patientenspezifischer als bisher angenommen. Zum Verständnis der zellbiologischen Abläufe (Signalling) und der bisher möglichen Prädiktion eines erfolgreichen Einsatzes von BMP sind in diesem Artikel die relevanten Erkenntnisse zusammengefasst.

\section{Probleme in der Behandlung von meta- und diaphysären Tibiafrakturen}

Trotz der stetigen Verbesserungen der operativen Versorgung von Frakturen kommt es im Bereich der langen Röhrenknochen nach wie vor zu Komplikationen, wie beispielsweise einer verzögerten Frakturheilung, Pseudarthrosen sowie Implantatversagen oder Infektionen. Insbesondere die verzögerte Frakturheilung stellt ein immanentes chirurgisches und aber auch volkswirtschaftliches Problem dar. Die Therapieoptionen aus der Sicht des Chirurgen sind limitiert, und die Heilungstendenz lässt sich nur schwer beeinflussen oder vorhersagen. Für den Patienten stellen sich eine verzögerte Rekonvaleszenz und Mehrfach-OPs ein. Volkswirtschaftlich ist die Wiedereingliederung in das Berufsleben und das Erlangen der Vollbeschäftigung weder zeitlich noch in Bezug auf den Umfang abzusehen. Die in der Literatur beschriebene Prävalenz von Tibiafrakturen mit verzögerter Frakturheilung variiert in Abhängigkeit des Weichteilschadens. Für die Gustilo-Ander-

\section{Abstract \\ $\nabla$}

In the therapy for pseudarthroses of the proximal tibia, the human recombinant bone morphogenetic proteins (BMP-2 and BMP-7) have been used for several years. Despite their limited and specified use as local mediators of bone healing, no conclusions regarding the therapeutic success can be made beforehand. The regulatory mechanisms have turned out to be much more complex and patient-specific than had been assumed before. To help understand the cell biological processes (signalling) and the current possibilities of predicting a successful use of BMP, this article summarises the relevant findings.
son-Grade I, II und IIIA [1] ist eine verzögerte Frakturheilung in 16 bis $60 \%$ der Fälle beschrieben, wohingegen die Grade IIIB und IIIC in 43 bis $100 \%$ verzögert heilen [2]. Knochen unterliegen einem kontinuierlichen Auf- und Abbau. Während dieser kontinuierlichen Modulierung sorgen die Osteoblasten und Osteozyten für den Gewebeaufbau und dessen Stabilität und die Osteoklasten für die Knochenresorption [3,4]. Nach einer Fraktur kann dadurch die Knochenmatrix wieder funktionsfähig hergestellt werden und verheilen. Störungen in diesem ausbalancierten System können zu schwerwiegenden Komplikationen führen, beispielsweise zu Osteopenie, Osteoporose oder im Falle der verzögerten Frakturheilung zu Pseudarthrosen [5-7].

Eine Möglichkeit der Pseudarthrosenbehandlung ist der Einsatz der Bone morphogenetic Proteins (BMPs). Die Behandlung wird nicht als Ersttherapie von den Krankenkassen bezahlt. Ziel dieser Übersichtsarbeit ist es, unter Zuhilfenahme klinischer Studien aus der Literatur diesen Punkt kritisch zu hinterfragen und zu beleuchten, ob 
ein frühzeitiger Einsatz der BMPs einen sozioökonomischen Vorteil bietet.

Dazu wurden in dieser Übersichtsarbeit folgende Studientypen berücksichtigt: (i) randomisiert kontrollierte Studien, (ii) Kosten-Effizienz-Analysen oder (iii) reine Kostenanalysen. Es wurden Studien unter Verwendung von BMP2 und BMP7 eingeschlossen. Als Interventionsarten wurden sowohl die Applikation von BMPs im Vergleich zur Osteosynthese mit und ohne Knochentransplantation als auch die Applikation von BMPs in Kombination mit Knochentransplantation im Vergleich zur alleinigen Knochentransplantation untersucht. Zum Patientenkollektiv zählten nur Personen mit akuten Knochenbrüchen oder Pseudarthrosen, die ein adultes Skelett und ein Mindestalter von 16 Jahren aufwiesen. Zum Ausschluss von Studien führte: (i) der Einschluss von Probanden mit ernsthaften Begleiterkrankungen oder (ii) eine Probandenzahl von unter 10. Aufgrund dieser Einbzw. Ausschlusskriterien fokussiert sich diese Übersichtsarbeit auf 15 Artikel, die zwischen 1999 und 2010 in den Datenbanken PubMed und Cochrane Library veröffentlicht wurden.

Als primäres „Outcome“ wurden die Zeit bis zur vollständigen Knochenheilung sowie die Knochenheilungsrate gezählt. Als sekundäres „Outcome“ wurde vor allem die Infektionsrate, aber auch die Anzahl der sekundären Interventionen, Implantatkomplikationen oder ungewollter Nebenwirkungen der BMPs berücksichtigt.

Für die Darstellung der Tierstudien sowie der BMP-Signaltransduktion und deren Regulierung wurde eine gesonderte Datenbanksuche durchgeführt.

\section{Frakturremodelling mit humanen rekombinanten BMPs \\ $\nabla$}

Die Bone morphogenetic Proteins (BMPs) gehören zur Transforming-Growth-Factor-beta-(TGF- $\beta$-)Superfamilie [8]. BMPs sind wichtige regulierende Faktoren im Zuge der Regeneration und Neubildung von Knochen [4]. Sie üben Einfluss auf die Menge neugebildeter Knochenmasse und die Geschwindigkeit ihrer Entstehung aus, sowohl während der Knochenheilung als auch während der normalen physiologischen Modulierung von Knochen [9]. BMPs sind daher immer mehr in den Mittelpunkt des Forschungsinteresses gerückt, um chirurgische Maßnahmen und Eingriffe zu verbessern.

\section{Erfolgreicher Einsatz von BMPs in Tierversuchen}

In ersten Tierversuchen wurde die biologische Aktivität der BMPs durch Überexpression mit viralen Konstrukten nachgewiesen. Dabei zeigten besonders BMP-2, -4, -6, -7 und -9 eine osteoinduktive Wirkung $[4,10]$. Daraufhin wurden erste BMPs rekombinant durch biotechnologische Methoden hergestellt (BMP-2, -4 und -7). Deren biologische Aktivität wurde in einer Vielzahl von Tiermodellen untersucht. Beispielsweise konnte durch das Aufbringen der rhBMPs auf Implantaten deren Einwachsen stark verbessert werden; so z. B. in den Studien von Fukunaga et al. [11] und Sumner et al. [12], die in einem Kaninchenmodell das Einwachsen von porösen Implantaten durch Beschichtung mit rhBMP-2 dosisabhängig verbessern konnten. Ähnliche Ergebnisse erzielten Thorey et al. [13,14], Schmidmaier et al. [15] und Itoh et al. [16] mit Titanimplantaten in Kaninchen und Ratte. Darüber hinaus konnte nach Gabe von rhBMPs die Bildung neuen Knochens an verschiedenen Stellen des Skeletts angeregt und so Knochendefekte kritischer Größe behandelt werden. Dies zeigt sich bereits früh in der Kaninchen-Studie von Woo et al. [17]. Diese Daten wurden durch weitere Studien an Ratten, Kaninchen oder Schafen bestätigt [18-23]. Aufgrund der Verwendung monogenetischer Tierlinien, einer oft streng definierten Altersgruppe (und Geschlecht) der Tiere sowie dem Fehlen von Nebenerkrankungen konnte in diesen Studien ein Schwerpunkt auf die geeignete Applikationsweise der rhBMPs gelegt werden, um eine mögliche klinische Anwendung voranzutreiben. So konnte durch Modifikationen z.B. gezielt Einfluss auf die Fibrinlöslichkeit des rhBMP [24] oder auf seine kontrollierte Freisetzung [25-27] genommen werden.

\section{Zulassungen humaner rekombinanter BMPs \\ für den klinischen Einsatz}

Aus der Familie der BMPs werden seit einigen Jahren bereits das BMP-2 und das BMP-7 klinisch zur Anwendung gebracht. Einsatz finden jeweils die rekombinanten humanen Proteine der BMPs. Erfolgreich den Weg zur Zulassung durchlaufen hat das rekombinante humane Protein BMP-2 (rhBMP-2). Das unter dem Arzneimittelnamen Dibotermin alfa vertriebene InductOs ${ }^{\circledR}$ der Firma Wyeth ist ein Kit, bestehend aus dem Proteinpulver, einem Lösungsmittel und einer bioabbaubaren Matrix aus Rinderkollagen. Das Lösen des Pulvers und Beträufeln der Matrix erfolgt während des chirurgischen Eingriffs und wird auf die frakturierte Region gelegt. InductOs findet Anwendung als Ergänzung nach Versagen der Standardtherapie zur Behandlung akuter Tibiafrakturen. Bei verzögerter Knochenbruchheilung bzw. nach Ausbildung einer Pseudarthrose wird in vielen Fällen zusätzlich zur Anlagerung von autologer Spongiosa oder Tricalciumphosphat BMP zur Unterstützung angewendet [28]. Ebenfalls zugelassen ist das rhBMP-7. Es wird als Eptotermin alfa unter dem Namen Osigraft ${ }^{\circledR}$ der Firma Stryker vertrieben. Das Protein liegt, an eine Kollagenmatrix gebunden, in Pulverform vor und ergibt nach Zugabe isotonischer Natriumchloridlösung zur Injektion eine Suspension, welche direkt in den Frakturbereich appliziert wird. Zugelassen ist Osigraft zur Behandlung von Tibiafrakturen, die eine Pseudarthrosenbildung aufweisen, über einen Zeitraum von mindestens 9 Monaten bestehen und bei denen Vorbehandlungen mittels autologer Transplantation fehlschlugen oder kontraindiziert sind [29].

Klinische Studien mit humanen rekombinanten BMPs Trotz immer neuer Erkenntnisse und der erfolgreichen Anwendung von rhBMPs an Menschen (Übersicht über klinische Studien siehe 0 Tab. 1) ist in der klinischen Praxis hinsichtlich des Ansprechens auf die Behandlung eine hohe Spendervariabilität zu erkennen.

Die wohl größte klinische Studie über den Einsatz von rhBMP-2 ist die sogenannte BESTT-Studie (BMP-2 Evaluation in Surgery for Tibial Trauma). In dieser prospektiven kontrollierten und randomisierten Studie mit 450 Patienten wurde der Einsatz von rhBMP-2 zur Behandlung von offenen Tibiafrakturen untersucht. Patienten mit offenen Tibiafrakturen wurden in der Studie zusätzlich zur intramedullären Stabilisierung mit $1,5 \mathrm{mg} / \mathrm{ml}$ rhBMP-2 behandelt [2]. Die Studie ergab, dass die Anwendung von rhBMP-2 die Anzahl von Folgeoperationen signifikant, um 44\% $(p=0,0005)$, reduzierte. Dies wird durch die Studien von Friedlaender et al. [30], McKee et al. [31], Jones et al. [32] und Calori et al. [33] mit einem deutlich kleineren Patientenkollektiv bestätigt, obwohl bei den Studien von Friedlaender et al. und Jones et al. die Infektionsrate durch Anwendung von rhBMPs stieg. In der BESTT-Studie konnte das Auftreten von Infektionen unter Ver- 
Tab. 1 Übersicht ausgewählter klinischer Studien mit BMPs.

\begin{tabular}{|c|c|c|c|c|c|c|c|}
\hline \multirow[t]{2}{*}{ Studie } & \multirow{2}{*}{$\begin{array}{l}\text { Patienten- } \\
\text { kollektiv }\end{array}$} & \multirow[t]{2}{*}{ Frakturart } & \multirow[t]{2}{*}{ Intervention } & \multicolumn{2}{|c|}{ Heilungsrate } & \multicolumn{2}{|l|}{ Infektionsrate } \\
\hline & & & & mit BMP & ohne BMP & mit BMP & ohne BMP \\
\hline $\begin{array}{l}\text { Cook } 1999 \\
{[55]}\end{array}$ & 30 & $\begin{array}{l}\text { Pseudarthrose der } \\
\text { Tibia }\end{array}$ & $\begin{array}{l}\text { intramedulläre Nagelung mit rhBMP-7 oder } \\
\text { autologem Transplantat }\end{array}$ & $85,7 \%$ & $93,8 \%$ & - & $6,3 \%$ \\
\hline $\begin{array}{l}\text { Geesink } \\
1999[56]\end{array}$ & 24 & $\begin{array}{l}\text { Fibulafraktur kritischer } \\
\text { Größe }\end{array}$ & $\begin{array}{l}\text { mit/ohne Osteotomie mit 2,5 mg rhBMP-7 (mit } \\
1 \mathrm{~g} \text { Rinderkollagen), } 2 \mathrm{ml} \text { demineralisierter } \\
\text { Knochen oder } 1 \mathrm{~g} \text { Rinderkollagen }\end{array}$ & $83,3 \%$ & $66,7 \%$ & k. A. & k. A. \\
\hline $\begin{array}{l}\text { Chen } 2000 \\
\text { [57] }\end{array}$ & 80 & $\begin{array}{l}\text { Pseudarthrose der } \\
\text { Tibia }\end{array}$ & $\begin{array}{l}\text { Intervention (nicht beschrieben) mit natürlichem } \\
\text { nichtorganischem Knochen/BMP oder Autograft- } \\
\text { Knochen oder andere operative Maßnahme (nicht } \\
\text { beschrieben) }\end{array}$ & $100 \%$ & $100 \%$ & k. A. & k. A. \\
\hline $\begin{array}{l}\text { Friedlaender } \\
2001[30]\end{array}$ & 122 & $\begin{array}{l}\text { Pseudarthrose der } \\
\text { Tibia }\end{array}$ & $\begin{array}{l}\text { intramedulläre Nagelung mit rhBMP-7 oder } \\
\text { Autograft-Knochen }\end{array}$ & $\begin{array}{l}61,9 \% \\
4,8 \% *\end{array}$ & $\begin{array}{c}73,8 \% \\
9,8 \% *\end{array}$ & $22,2 \%$ & $19,7 \%$ \\
\hline \multirow[t]{2}{*}{$\begin{array}{l}\text { Govender } \\
2002[58]\end{array}$} & \multirow[t]{2}{*}{450} & \multirow[t]{2}{*}{$\begin{array}{l}\text { akute offene Fraktur } \\
\text { hauptsächlich der Dia- } \\
\text { physe }\end{array}$} & $\begin{array}{l}\text { intramedulläre Nagelung mit/ohne } 0,75 \mathrm{mg} / \mathrm{ml} \\
\text { rhBMP-2 }\end{array}$ & $37 \%^{*}$ & \multirow[t]{2}{*}{$46 \% *$} & $\begin{array}{l}15 \%(G A I+I I) ; \\
29 \%(G A I I I A+ \\
\text { IIIB) }\end{array}$ & $\begin{array}{l}15 \%(G A I+ \\
\text { II); } 44 \%(G A \\
I I I A+I I B)\end{array}$ \\
\hline & & & $\begin{array}{l}\text { intramedulläre Nagelung mit/ohne } 1,5 \mathrm{mg} / \mathrm{ml} \\
\text { rhBMP-2 }\end{array}$ & $26 \% *$ & & $\begin{array}{l}21 \%(G A I+I I) \\
24 \%(G A I I I A+ \\
I I I B)\end{array}$ & \\
\hline $\begin{array}{l}\text { Maniscalco } \\
2002[59]\end{array}$ & 14 & $\begin{array}{l}\text { geschlossene Tibia- } \\
\text { fraktur }\end{array}$ & externe Fixierung mit oder ohne rhBMP-7 & k. A. & k. A. & k. A. & k. A. \\
\hline $\begin{array}{l}\text { McKee } 2002 \\
{[31]}\end{array}$ & 124 & offene Tibiafraktur & intramedulläre Nagelung mit oder ohne rhBMP-7 & $12,9 \%^{*}$ & $27,4 \% *$ & k. A. & k. A. \\
\hline $\begin{array}{l}\text { Jones } 2006 \\
{[32]}\end{array}$ & 30 & $\begin{array}{l}\text { Frakturen der Tibiadia- } \\
\text { physe }\end{array}$ & $\begin{array}{l}\text { Rekonstruktion der Tibia mit } 1,5 \mathrm{mg} / \mathrm{ml} \text { rhBMP- } \\
2(12 \mathrm{mg} \text { total) oder autogenem Knochen- } \\
\text { transplantat }\end{array}$ & $13,3 \%^{*}$ & $33,3 \%^{*}$ & $20 \%$ & $6,7 \%$ \\
\hline $\begin{array}{l}\text { Calori } 2006 \\
{[33]}\end{array}$ & 29 & $\begin{array}{l}\text { Pseudarthrose oder Frak- } \\
\text { tur kritischer Größe von } \\
\text { langen Röhrenknochen }\end{array}$ & $\begin{array}{l}\text { intramedulläre Nagelung, Platte oder externe } \\
\text { Fixierung mit } 3,5 \mathrm{~g} / 7 \mathrm{~g} \text { rhBMP-7 oder } 10 \mathrm{ml} / \\
20 \mathrm{ml} \text { thrombozytenreiches Plasma (PRP) }\end{array}$ & $\begin{array}{c}93,8 \% \\
6,3 \%\end{array}$ & $\begin{array}{l}61,5 \% \\
23,1 \% *\end{array}$ & k. A. & k. A. \\
\hline $\begin{array}{l}\text { Alt } 2006 \\
{[40]}\end{array}$ & 291 & $\begin{array}{l}\text { akute Fraktur Tibia- } \\
\text { schaft }\end{array}$ & $\begin{array}{l}\text { intramedulläre Nagelung mit/ohne } 1,5 \mathrm{mg} / \mathrm{ml} \\
\text { rhBMP-2 }\end{array}$ & \multicolumn{2}{|c|}{ nur Kostenanalyse } & $16,6 \%$ & $16,4 \%$ \\
\hline $\begin{array}{l}\text { Ekrol } 2008 \\
{[34]}\end{array}$ & 30 & $\begin{array}{l}\text { korrigierende Osteoto- } \\
\text { mie nach Fehlheilung }\end{array}$ & $\begin{array}{l}\text { externe } o \text {. interne Fixierung mit rhBMP-7 oder } \\
\text { autogenem Transplantat }\end{array}$ & $71,4 \%$ & $93,8 \%$ & $25 \%$ & $50 \%$ \\
\hline
\end{tabular}

* sek. Intervention notwendig zur Heilung; k. A. - keine Angabe

wendung von rhBMP-2 im Vergleich zur Kontrollgruppe signifikant verringert werden [2]. Dadurch konnte die durchschnittliche Heilungsdauer der Tibiafrakturen in der mit rhBMP-2-behandelten Gruppe im Durchschnitt um 39 auf 145 Tage reduziert werden. Es wurde der Schluss gezogen, dass der zu erwartende Erfolg einer rhBMP-2-Therapie mit dem Schweregrad der Fraktur bzw. des Weichteilschadens (Gustilo-Anderson-Klassifikation) zunimmt. Im Gegensatz zur BESTT-Studie reduzierte sich jedoch in der Studie von Ekrol et al. der Heilungserfolg durch den Einsatz von rhBMP-7 [34]. In weiteren Studien konnten darüber hinaus unvorhersehbare Sicherheitsrisiken der Therapie, wie z. B. Osteolysen an der Wirbelsäule, ektopische Knochenbildung, Radikulitis oder zervikale Weichteilschwellungen, beobachtet werden $[35,36]$. In 36\% der kombinierten Therapie mit BMP kam es dennoch zu keiner signifikanten Steigerung der sekundär knöchernen Durchbauung [37]. Die Ursachen für diese hohe Zahl an Nonoder Lowrespondern ist bisher nicht bekannt. Welche Rolle das Alter der Patienten, mögliche Nebenerkrankungen, deren Medikation und der hochvariable genetische Hintergrund dabei spielen, wurde bisher wenig untersucht. Auch werden rhBMPs entsprechend ihrer klinischen Zulassung erst nach strenger Indikationsstellung eingesetzt - meist erst, wenn bisherige Standardtherapien versagen und eine Transplantation autologen Knochens bereits ohne Erfolg durchgeführt wurde oder kontraindiziert ist. Möglicherweise sind zu diesem Zeitpunkt die Zielzellen der Therapie bereits zu geschädigt, um an die Erfolge der Tierversuche nahtlos anzuschließen. Dies zeigt deutlich, dass weitere prospek- tive, randomisierte Studien nötig sind, um den ggf. früheren Einsatz und die damit verbundene Effizienz der rhBMPs im klinischen Alltag aufzuzeigen und damit die Standardtherapie weiter zu optimieren [2].

\section{Kosten und Nutzen der Therapie mit rhBMPs}

Die verzögerte Frakturheilung verursacht Schmerzen und eine Einschränkung der Funktion. Neben einer Verminderung der Lebensqualität kommt es zur Verlängerung der Arbeitsunfähigkeit [2]. Die verzögerte Frakturheilung ist somit ein sozioökonomisches Problem. Eine Kosten-Nutzen-Analyse, welche die indirekten Kosten mit einschließt, könnte somit die Verwendung von rhBMPs in ein pharmakoökonomisch besseres Licht rücken. Die momentane Datenlage ist bisher sehr spärlich, und es steht nur eine vollständige Kosten-Nutzen-Analyse aus Großbritannien zur Verfügung [38]. Diese kommt, in Übereinstimmung mit der BESTT-Studie, zu dem Ergebnis, dass eine frühzeitige Anwendung von rhBMP-2 ökonomisch von Vorteil ist und in Abhängigkeit des Schweregrads der Fraktur steht (Gustilo-Anderson-Grad IIIB und IIIC) [39]. Um eine weitgehende Kostenvereinheitlichung, und damit eine Kostenminimierung, zu erzielen, wurde in Deutschland im Rahmen der Kostenerstattung durch die Krankenkassen im Jahr 2003 das System der Diagnosis related Group (DRG) eingeführt. In diesen Festbeträgen ist zum jetzigen Zeitpunkt die Erstattung einer Therapie mit rhBMP-2 nicht vorgesehen. Im Krankheitsfall sind die Krankenversicherungen verpflichtet, 12 Monate lang Krankengeld zu leisten, nachdem die ersten 6 Wo- 
Tab. 2 Übersicht weiterer Regulatoren der BMP-Signalkaskade.

\begin{tabular}{|c|c|c|}
\hline Regulator & $\begin{array}{l}\text { Effekt auf die BMP-ver- } \\
\text { mittelte Osteogenese }\end{array}$ & Wirkungsweise \\
\hline Noggin & Inhibition & $\begin{array}{l}\text { Noggin wird von Osteoblasten exprimiert und durch BMPs induziert (negativer Feedback-Mechanismus). Noggin } \\
\text { bindet extrazellulär BMP-2, }-4 \text { und }-7 \text {, sodass deren Rezeptorbindung verhindert wird. }\end{array}$ \\
\hline Chordin & Inhibition & Chordin erkennt und bindet BMP-2, -4 und -7 , sodass deren Rezeptorbindung verhindert wird. \\
\hline Gremlin & Inhibition & Gremlin erkennt und bindet BMP-2, -4 und -7 , sodass deren Rezeptorbindung verhindert wird. \\
\hline Follistatin & Inhibition & Follistatin antagonisiert BMP durch Komplexbindung mit BMP und BMP-Rezepor. \\
\hline BAMBI & Inhibition & $\begin{array}{l}\text { BAMBI, ein sogenannter Lockvogel-Rezeptor, ist ein Pseudo-Typ-I-Rezeptor, dem die Serin-Threonin-Kinase-Domäne } \\
\text { fehlt, und der somit nach Bindung von BMPs die Aktivierungskaskade nicht weiterleiten kann (negativer Feedback- } \\
\text { Mechanismus) [37]. }\end{array}$ \\
\hline Endofin & Verstärkung & $\begin{array}{l}\text { Endofin reguliert die Phosphorylierung von Alks. Es bindet Smad1 und fördert so dessen Phosphorylierung. Endofin } \\
\text { bindet auch Smad4 und präsentiert es Smad1 und -2 [60]. }\end{array}$ \\
\hline $\begin{array}{l}\text { I-Smads } \\
\text { (Smad6 und -7) }\end{array}$ & Inhibition & $\begin{array}{l}\text { Zur Gruppe der I-Smads (Inhibitory Smads) gehören Smad6 und -7. Sie können die Aktivierung der R-Smads durch } \\
\text { Bindung an den Typ-I-Rezeptor verhindern. Darüber hinaus können I-Smads durch direkte Bindung an aktivierte R- } \\
\text { Smads deren Komplexbindung mit Smad4 verhindern. Die I-Smads werden durch Typ-I-Rezeptor-Phosphorylierung } \\
\text { rekrutiert und stehen somit im Zusammenhang mit einem negativen Feedback-Mechanismus. Während sich die in- } \\
\text { hibierende Funktion von Smad6 eher auf die BMP-Signaltransduktion auswirkt, hemmt Smad7 den TGF- } \beta-\text { - Activin- } \\
\text { und BMP-Signalweg. }\end{array}$ \\
\hline BMP-3 & Inhibition & $\begin{array}{l}\text { BMP-3 fördert die Proliferation von mesenchymalen Zellen, verhindert aber deren Differenzierung (negativer Feed- } \\
\text { back-Mechanismus) }[4,61] \text {. }\end{array}$ \\
\hline \multirow[t]{2}{*}{ Smurf-1 und -2} & Inhibition & $\begin{array}{l}\text { Smad Ubiquitination regulatory Factors-1 und -2 (Smurf-1 und -2) erkennen Smad7 im Zellkern und bewirken dessen } \\
\text { Efflux in das Zytoplasma [45, 47]. Dadurch wird der Abbau (durch Ubiquitinierung) von phosphorylierten und nicht } \\
\text { phosphorylierten R-Smads gefördert [3], um die Menge an verfügbaren Signalproteinen im Grundzustand der Zelle } \\
\text { niedrig zu halten [48]. }\end{array}$ \\
\hline & Verstärkung & $\begin{array}{l}\text { Smurf-Proteine sind auch in der Lage, Komplexe mit sich im Zellkern befindenden R-Smads zu bilden und dann tran- } \\
\text { skriptionelle Repressoren zu erkennen und deren negativen Einfluss auf die Gentranskription zu unterbinden [49]. }\end{array}$ \\
\hline SKI-Oncogen & Inhibition & transkriptionelle Repression der BMP-induzierten Genexpression \\
\hline SnoN & Inhibition & transkriptionelle Repression der BMP-induzierten Genexpression \\
\hline $\begin{array}{l}\text { kanonische Wnt- } \\
\text { Signalkaskade }\end{array}$ & Verstärkung & fördert die Translokation von R-Smads in den Zellkern \\
\hline Sclerostin & Inhibition & $\begin{array}{l}\text { Sclerostin hemmt die Osteoblastenaktivität und -differenzierung. Sclerostin begünstigt die Apoptose von Osteo- } \\
\text { blasten. Sclerostin fungiert als indirekter Antagonist der BMP-Aktivität durch Inhibieren des Wnt-Signalwegs. }\end{array}$ \\
\hline $\begin{array}{l}\text { Dickkopf (DKK) } \\
1 \text { und } 2\end{array}$ & Inhibition & $\begin{array}{l}\text { DKK1 und DKK2 hemmen die Osteoblastenaktivität und -differenzierung. Sie fungieren als indirekte Antagonisten } \\
\text { der BMP-Aktivität durch Inhibieren des Wnt-Signalwegs. }\end{array}$ \\
\hline
\end{tabular}

chen über den Arbeitgeber und dessen jeweilige Versicherung beglichen wurden [40]. Die durchschnittliche Heilungsdauer von Tibiafrakturen im Rahmen der BESTT-Studie dauerte sowohl in der Kontrollgruppe (184 Tage) als auch in der mit $1,5 \mathrm{mg} / \mathrm{ml}$ rhBMP-2 behandelten Gruppe (145 Tage) länger als 6 Wochen. Die in dieser Studie beobachtete Differenz von 39 Tagen wird somit zu den indirekten Kosten gezählt, welche durch die Krankenversicherung bezahlt werden müssen. Allerdings ergab die Studie auch, dass durch den Einsatz von rhBMP-2 nicht nur die Heilungsdauer der Fraktur, sondern auch die Menge an notwendigen sekundären Interventionen sowie die Infektionsrate signifikant reduziert wurden [2]. Da vor allem diese sekundären Interventionen, wie z.B. die Re-Osteosynthesen oder die autologe Knochentransplantation, mit hohen direkten und indirekten Kosten verbunden sind, würde deren vermindertes Auftreten die Folgekosten signifikant reduzieren. Bisherige Untersuchungen hinsichtlich der Frakturheilung ergaben, dass der Einsatz der rhBMPs keinen zusätzlichen therapeutischen Erfolg bringt und somit als gleichwertig anzusehen ist. Der Vorteil liegt jedoch in der Vermeidung des operativen Eingriffs zur Entnahme autologen Knochens und erspart dem Patienten alle damit verbundenen Komplikationen und notwendigen sekundären Interventionen $[30,38]$.

\section{Molekularbiologische Betrachtung des Therapieversagens \\ $\nabla$}

Als Zytokine sind die BMPs in der Lage, das Wachstum und die Differenzierung von Zellen zu regulieren [41], wobei die jeweilige Funktion von der Isoform des Zytokins definiert wird. So ist beispielsweise BMP-3 bekannt dafür, das Wachstum von Osteoblasten zu fördern, während BMP-2, -4, -6, -7 und -9 deren Differenzierung fördern. Von entscheidender Bedeutung ist dabei die Aktivierung von Signalkaskaden und die Weiterleitung der jeweiligen Signale ins Zellinnere, um dort den regulierenden Einfluss auf die Transkription von Zielgenen und damit einhergehend die Zellfunktionen ausüben zu können. Jeder dieser Schritte kann durch körpereigene Faktoren unterstützt oder verhindert werden (Übersicht siehe $\bullet$ Tab. 2), was wiederum einen Einfluss auf die Osteogenese hat. Im Einzelnen sind dies:

\section{BMP-Rezeptorbindung}

Hierzu binden die BMP-Moleküle an einen in der Zellmembran verankerten Rezeptor. Für die Bindung und Signalweiterleitung der Signalproteine aus der TGF- $\beta$-Superfamilie stehen 2 Typen von Rezeptoren zur Verfügung, welche zur Klasse der SerinThreonin-Kinasen gehören [4]. Zum einen handelt es sich um die Typ-I-Rezeptoren, bestehend aus 7 verschiedenen Rezeptoren namens Activin-Receptor-like Kinase (Alk) 1-7 [41] und zum anderen um die 4 Typ-II-Rezeptoren: TGFR-II, BMPR-II, 


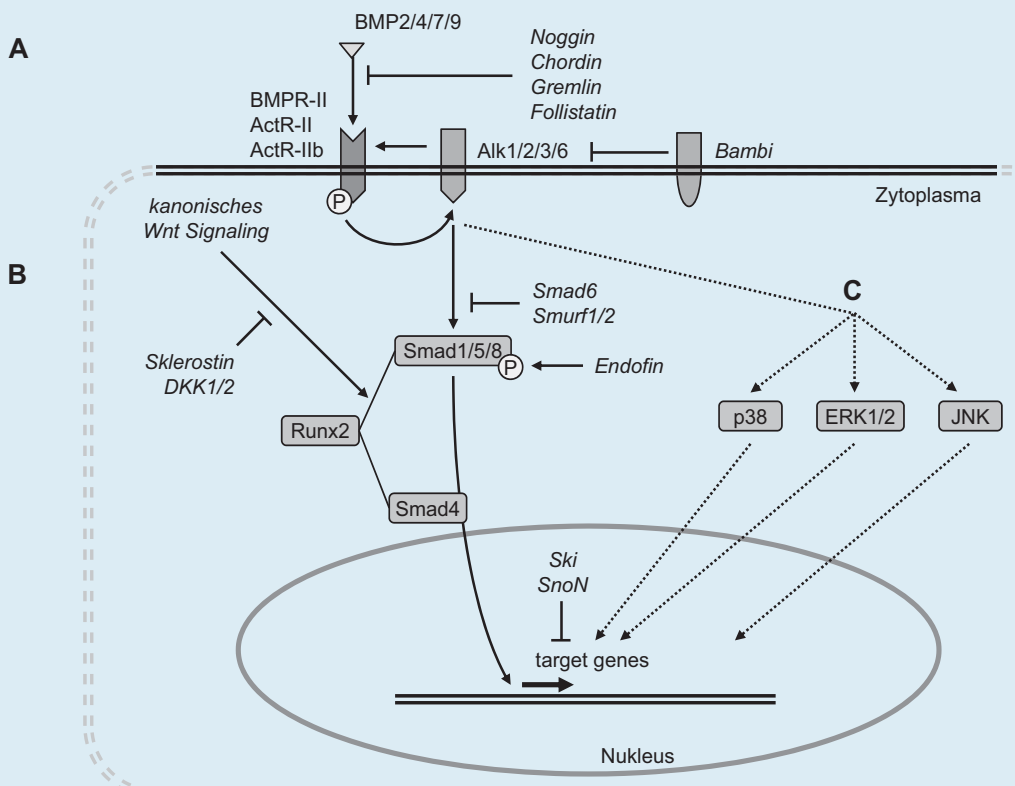

ActR-II und ActR-IIb. Die BMPs vermitteln ihre Signale lediglich über Alk1, Alk2, Alk3 und Alk6 der Typ-I-Rezeptoren sowie über BMPR-II, ActR-II und ActR-IIb der Typ-II-Rezeptoren. Nach der Bindung des BMP-Liganden an einen Typ-I-Rezeptor wird ein intrazellulärer Zusammenschluss mit dem entsprechenden Typ-IIRezeptor ausgelöst. Die konstitutiv phosphorylierten Typ-II-Rezeptoren sind nun in der Lage, den Typ-I-Rezeptor durch die Übertragung des Phosphatrests zu aktivieren (phosphorylieren) $[3,42,43]$ ( Abb. 1 a). Bereits dieser Schritt kann durch Bindung des BMP-Liganden an extrazelluläre Inhibitoren, wie z.B. Noggin, Chordin, Gremlin und Follistatin oder an das membrangebundene BMP and Activin Receptor Membrane bound Inhibitor (BAMBI), einem sogenannten Lockvogel-Rezeptor, unterbrochen werden, was die Inhibierung der Osteogenese zur Folge hat.

\section{Smad-abhängige Signalweiterleitung in der Zelle}

Der durch Phosphorylierung aktivierte Typ-I-Rezeptor erkennt die zytoplasmatischen rezeptorregulierten Smad-Proteine (RSmads). Nach derzeitigem Wissensstand werden von den 5 vorkommenden R-Smads (1, 2, 3, 5 und 8) R-Smad 1, -5 oder -8 durch die BMPs phosphoryliert [42]. Das Common-Mediator-SmadProtein (co-Smad, Smad4) erkennt die aktivierten R-Smads und bildet mit ihnen einen heterodimeren oder -trimeren Komplex. Durch diese Assoziation ist die Translokation in den Nukleus möglich [44]. Die R-Smad-Smad-4-Komplexe akkumulieren daraufhin im Nukleus, erkennen für die Expression bestimmter Zielgene verantwortliche Strukturen (Promotoren) und regulieren somit die Transkription dieser Gene, sowohl positiv als auch negativ [45-47] ( $\bigcirc$ Abb. 1 b). Die wohl wichtigste Erkennungssequenz für die BMP-Signaltransduktion stellt das Smad binding Element (SBE) dar [44]. Der Schritt der Signalweiterleitung wird durch verschiedene zelleigene Regulationsmechanismen beeinflusst. Eine Verstärkung der Signalweiterleitung wird z.B. durch Endofin erlangt. Durch dessen Bindung an Smad1 wird dieses vermehrt phosphoryliert und seine Komplexbildung mit Smad4 gefördert. Ein gegenteiliger Effekt wird durch die I-Smads (Inhibitory Smads), bestehend aus Smad6 und -7 , erzielt. Dabei wirkt insbesondere Smad6 inhibitorisch auf die BMP-Signalweiterlei-
Abb. 1 a bis c Schematische Darstellung der BMPSignalweiterleitung: a BMP-Rezeptorbindung und folgende b Smad-abhängige und c Smad-unabhängige Signalweiterleitung in den Zellkern. Regulatoren des Signalwegs sind kursiv eingezeichnet. tung. Der aktive Abbau von sich im Zytoplasma befindenden phosphorylierten und nicht phosphorylierten R-Smads wird von den Smad Ubiquitination regulatory Factors-1 und -2 (Smurf-1 und -2 ) durch Ubiquitinierung induziert $[3,48]$. Allerdings sind die Smurf-Proteine auch dazu in der Lage, Komplexe mit nukleären (sich im Zellkern befindlichen) R-Smads zu bilden und dann transkriptionelle Repressoren, wie z.B. das SKI-Oncogen und SnoN (ski like oncogen), zu erkennen und deren negativen Einfluss auf die Gentranskription zu unterbinden [49]. Darüber hinaus lassen bisherige Untersuchungen vermuten, dass $\beta$-Catenin, ein Signalprotein in der Wnt-Signalkaskade, die Translokation aktivierter R-Smads in den Zellkern reguliert [50]. Auf diesem Wege können Inhibitoren wie Sclerostin (SOST) und Dickkopf-1 und -2 (DKK1/2), als direkte Inhibitoren des Wnt-Signalwegs nachgeordnet die BMP-Signaltransduktion verhindern. Ähnlich dem Wnt-Signalweg werden der Fibroblast Growth Factor (FGF) Signaltransduktion und der Notch-Signalkaskade synergistische Effekte auf die BMP-vermittelte Osteogenese zugeschrieben [3].

\section{Smad-unabhängige Signalweiterleitung in der Zelle}

Neben der direkten Aktivierung der Smad-Signalkaskade existieren auch Smad-unabhängige Wege der Signalweiterleitung nach Bindung der BMPs an die jeweiligen Rezeptoren. Dabei sind die mitogen aktivierten Proteinkinasen (MAPK) von entscheidender Bedeutung. Im Rahmen der Smad-unabhängigen BMP-Signaltransduktion spielen die MAPK Extracellular Signal-related Kinases 1/2 (ERK1/2) und p38 die größte Rolle. Die MAPK werden durch extrazelluläre Stimuli, beispielsweise auch durch Bindung von BMPs an deren Rezeptoren, aktiviert. Hierzu wird erst eine Kaskade bestehend aus vorgeschalteter MAP-Kinase-Kinase-Kinase (MAPKKK) und MAP-Kinase-Kinase (MAPKK) durchlaufen, bevor die MAPK dann in der Lage sind, die Genexpression mit zu regulieren und in verschiedene zelluläre Vorgänge, wie z.B. Wachstum, Differenzierung und Apoptose, einzugreifen [51] ( Abb. 1 c). Die genaue Funktion und der Mechanismus, den die MAPK im Rahmen der osteogenen Differenzierung und Knochenentstehung ausführen, sind bisher noch nicht bekannt. Es wird vermutet, dass die unterschiedlichen MAPK verschiedenartige 
Effekte auf die BMP-vermittelte Osteogenese ausüben [52]. Erst kürzlich veröffentlichte eine chinesische Forschergruppe, dass die Inhibierung von p38 zu einem signifikanten Rückgang der BMP-induzierten osteogenen Differenzierung mesenchymaler Vorläuferzellen führt, wohingegen die Hemmung von ERK1/2 zu einer gesteigerten Osteogenese führt [52].

\section{Übersetzung der BMP-Signale}

Die BMP-assoziierten Signale, sowohl Smad-abhängig als auch Smad-unabhängig vermittelt, laufen im Zellkern zusammen und regulieren dort die Transkription verschiedener Zielgene. Eine entscheidende Rolle für die richtige Interpretation der BMP-assoziierten Signale spielt dabei das korrekte Zusammenspiel zwischen lokaler Aktivität und Bindungsaffinität von spezifischen Kofaktoren. Diese können die Transkription der Zielgene sowohl fördern als auch hemmen. Runt-related Transcription Factor 2 (Runx2) ist einer der wichtigsten, die Osteogenese regulierenden Transkriptionsfaktoren. Runx2 kann direkt mit den BMP-aktivierten Smad-Proteinen interagieren und so die Expression osteoblastenspezifischer Gene induzieren. Ein weiterer, für die Reifung von Osteoblasten und Knochenbildung essenzieller Transkriptionsfaktor ist Osterix. Entgegen älterer Berichte, wo Osterix dem Runx2 nachgeschaltet ist, geben neueste Erkenntnisse Hinweis darauf, dass die Expression von Osterix, zumindest im Rahmen der BMP-2-Signaltransduktion, hauptsächlich über Dlx5 mediiert wird, und nicht über Runx2 [3].

\section{Klinischer Ausblick}

Dies zeigt, dass die BMP-Signaltransduktion auf molekularer Ebene durch eine Vielzahl von direkten und indirekten Inhibitoren unterbrochen werden kann. Aufgrund der interindividuellen Unterschiede der Patienten ist es schwer, einen einzigen Regulator für das Versagen der Therapie verantwortlich zu machen. Den wahrscheinlich maßgeblichsten Anteil hinsichtlich des Eingriffs in die BMP-Signaltransduktion stellen potenziell Veränderungen auf Transkriptionsebene dar. Welche Rolle das Alter der Patienten oder mögliche Nebenerkrankungen dabei spielen, wurde bisher vernachlässigt, obwohl diese Faktoren neben dem variablen genetischen Hintergrund die entscheidenden Unterschiede zum Tiermodell darstellen [18-23]. So existieren Hinweise, dass einige BMP-Inhibitoren durch chronische Entzündungsreaktionen vermehrt exprimiert werden. So konnten bei Patienten mit chronischen Erkrankungen, wie z. B. einer Leberfibrose und -zirrhose oder einem Diabetes mellitus, vermehrt aktives TGF- $\beta_{1}$ im Serum nachgewiesen werden. In ausgereiften Osteozyten wird durch TGF- $\beta$ die Expression von Sclerostin angeregt, um eine überschüssige Differenzierung von Osteoblasten zu verhindern [53]. Wir selbst konnten zeigen, dass die chronische Exposition mit diesem Zytokin die Aktivität und Differenzierung von Osteoblasten-Vorläuferzellen, durch Inhibierung des BMP-Signalwegs, verhindert [5]. Dies geschah durch die vermehrte Expression des transkriptionellen Regulators SnoN. Durch SnoN wurden vermehrt Histon-Deacetylasen (HDACs) rekrutiert und aktiviert, welche die Expression für die Signaltransduktion notwendiger Faktoren, wie z.B. Smad1 und Alk1, beeinträchtigten [37]. So könnte die lokale Applikation von HDAC-Inhibitoren die BMPSignalweiterleitung möglicherweise fördern und auf diesem Weg die Frakturheilung fördern. Unter Betrachtung der unterschiedlichen BMP-Signaltransduktionskaskaden gibt es jedoch weitere potenzielle Strukturen und Prozesse, welche die Signal- weiterleitung unterbinden und somit zu einem ausbleibenden Therapieerfolg führen können.

Bereits die Bindung der BMPs an die Rezeptoren kann durch verschiedene Inhibitoren oder konkurrierende Zytokine unterbunden werden. So versucht die Arbeitsgruppe um McMahon [54] beispielsweise, durch gezielten „knock-down“ konkurrierender BMPs, wie z.B. dem BMP-3, die Osteogenese zu fördern. Alternativ könnte durch eine veränderte biotechnologische Herstellung der rekombinanten humanen BMPs deren Struktur so verändert werden, dass sie von lokalen Inhibitoren, wie z.B. Noggin, Chordin, Gremlin, Follistatin oder BAMBI nicht mehr erkannt werden. Auch die Herstellung alternativer Isoformen der rekombinanten humanen BMPs, wie z.B. des BMP-6 oder des BMP-9, könnte zu einer effizienteren Therapie führen. So konnte in ersten Tierstudien gezeigt werden, das die Expression von BMP-9 die Osteogenese signifikant verbesserte [50]. Weiterhin wurde gezeigt, dass die durch BMP-9 vermittelte Signaltransduktion nicht identisch zu der von BMP-2 und BMP-7 ist. Hervorzuheben ist dabei, dass im Gegensatz zu den beiden bereits klinisch eingesetzten BMPs keine Inhibierung durch das körpereigene Noggin stattfindet [9] und BMP-9 auch nicht durch BMP-3 inhibiert wird [4]. Aufgrund dieser Ergebnisse ist es vorstellbar, dass ein rh-BMP-9 in der klinischen Anwendung effektiver ist als rh-BMP-2 oder rh-BMP-7. Allerdings sind weitere Untersuchungen notwendig, um die genauen Mechanismen von rh-BMP-9 aufzudecken.

Zusammenfassend konnten wir zeigen, dass für Pseudarthrosen mit einem begleitenden Weichteilschaden Gustilo-Anderson Grad IIIB und IIIC die BMP-Applikation Vorteile in der Behandlung bringt. Eine Anpassung des DRG-Systems unter der Berücksichtigung der Verwendung von BMPs könnte nicht nur zu vermeintlich sozioökonomischen Einsparungen führen, sondern auch die Lebensqualität des einzelnen Patienten deutlich verbessern. Aus der dargelegten Datenlage lässt sich somit die Schlussfolgerung ziehen, dass ein frühzeitiger Einsatz der BMPs zur Behandlung von Pseudarthrosen in Abhängigkeit des Schweregrads der Fraktur sowie des Weichteilschadens potenzielle Vorteile für den Patienten, und infolge auch für das Gesundheitssystem, bringen kann.

\section{Interessenkonflikt: Nein}

\section{Literatur}

1 Strohm PC, Bannasch H, Helwig P et al. Offene Fraktur und Weichteilschaden. Z Orthop Unfall 2010: 148: 95-112

2 Govender S, Csimma C, Genant HK et al. Recombinant human bone morphogenetic protein 2 for treatment of open tibial fractures: a prospective, controlled, randomized study of four hundred and fifty patients. J Bone Joint Surg Am 2002; 84: 2123-2134

3 Chen G, Deng C, Li YP. TGF-beta and BMP signaling in osteoblast differentiation and bone formation. Int J Biol Sci 2012; 8: 272-288

4 Kang $Q$ Sun M, Cheng $H$ et al. Characterization of the distinct orthotopic bone-forming activity of 14 BMPs using recombinant adenovirus-mediated gene delivery. Gene Ther 2004; 11: 1312-1320

5 Ehnert S, Baur J, Schmitt A et al. TGF-beta1 as possible link between loss of bone mineral density and chronic inflammation. PLoS One 2010; 5: e14073

6 Dobele S, Horn C, Eichhorn S et al. The dynamic locking screw (DLS) can increase interfragmentary motion on the near cortex of locked plating constructs by reducing the axial stiffness. Langenbecks Arch Surg 2010; 395: 421-428

7 Perren SM, Perren T, Schneider E. Biologie und Osteosynthese - Ein Widerspruch? Ther Umsch 2003; 60: 713-721

8 Wozney JM, Rosen V, Celeste AJ et al. Novel regulators of bone formation: molecular clones and activities. Science 1988; 242: 1528-1534

9 Rosen V. BMP and BMP inhibitors in bone. Ann N Y Acad Sci 2006; 1068: $19-25$ 
10 Breitbart AS, Grande DA, Mason JM et al. Gene-enhanced tissue engineering: applications for bone healing using cultured periosteal cells transduced retrovirally with the BMP-7 gene. Ann Plast Surg 1999; 42: 488-495

11 Fukunaga K, Minoda Y, Iwakiri $K$ et al. Early biological fixation of porous implant coated with paste-retaining recombinant bone morphogenetic protein 2. J Arthroplasty 2012; 27: 143-149

12 Sumner DR, Turner TM, Urban RM et al. Locally delivered rhBMP-2 enhances bone ingrowth and gap healing in a canine model. J Orthop Res 2004; 22: 58-65

13 Thorey F, Menzel H, Lorenz C et al. Enhancement of endoprosthesis anchoring using BMP-2. Technol Health Care 2010; 18: 217-229

14 Thorey F, Menzel H, Lorenz C et al. Osseointegration by bone morphogenetic protein- 2 and transforming growth factor beta2 coated titanium implants in femora of New Zealand white rabbits. Indian J Orthop 2011; 45: 57-62

15 Schmidmaier G, Wildemann B, Cromme F et al. Bone morphogenetic protein-2 coating of titanium implants increases biomechanical strength and accelerates bone remodeling in fracture treatment: a biomechanical and histological study in rats. Bone 2002; 30: 816-822

16 Itoh S, Matubara M, Kawauchi T et al. Enhancement of bone ingrowth in a titanium fiber mesh implant by rhBMP-2 and hyaluronic acid. J Mater Sci Mater Med 2001; 12: 575-581

17 Woo BH, Fink BF, Page R et al. Enhancement of bone growth by sustained delivery of recombinant human bone morphogenetic protein-2 in a polymeric matrix. Pharm Res 2001; 18: 1747-1753

18 Little DG, McDonald M, Bransford R et al. Manipulation of the anabolic and catabolic responses with OP- 1 and zoledronic acid in a rat critical defect model. J Bone Miner Res 2005; 20: 2044-2052

19 Pluhar GE, Turner AS, Pierce AR et al. A comparison of two biomaterial carriers for osteogenic protein-1 (BMP-7) in an ovine critical defect model. J Bone Joint Surg Br 2006; 88: 960-966

20 Patel ZS, Young S, Tabata Y et al. Dual delivery of an angiogenic and an osteogenic growth factor for bone regeneration in a critical size defect model. Bone 2008; 43: 931-940

21 Donati D, Di Bella C, Lucarelli E et al. OP-1 application in bone allograft integration: preliminary results in sheep experimental surgery. Injury 2008; 39: 65-72

22 Wildemann B, Lange K, Strobel C - et al. Local BMP-2 application can rescue the delayed osteotomy healing in a rat model. Injury 2011; 42: $746-752$

23 Notodihardjo FZ, Kakudo N, Kushida $S$ et al. Bone regeneration with BMP-2 and hydroxyapatite in critical-size calvarial defects in rats. J Craniomaxillofac Surg 2012; 40: 287-291

24 Schmoekel HG, Weber FE, Schense JC et al. Bone repair with a form of BMP-2 engineered for incorporation into fibrin cell ingrowth matrices. Biotechnol Bioeng 2005; 89: 253-262

25 Chung YI, Ahn KM, Jeon SH et al. Enhanced bone regeneration with BMP-2 loaded functional nanoparticle-hydrogel complex. J Control Release 2007; 121: 91-99

26 Strobel C, Bormann N, Kadow-Romacker A et al. Sequential release kinetics of two (gentamicin and BMP-2) or three (gentamicin, IGF-I and BMP-2) substances from a one-component polymeric coating on implants. J Control Release 2011; 156: 37-45

27 Wildemann B, Kandziora F, Krummrey $G$ et al. Local and controlled release of growth factors (combination of IGF-I and TGF-beta I, and BMP-2 alone) from a polylactide coating of titanium implants does not lead to ectopic bone formation in sheep muscle. J Control Release 2004; 95: 249-256

28 Dibotermin alfa (InductOs ${ }^{\circledR}$, Wyeth). In: Pharmazeutische Zeitung online; Govi-Verlag (2003). Im Internet: http://www.pharmazeutischezeitung.de/index.php?id=157; Stand: 25.03.2013

29 Eptotermin alfa, Osigraft ${ }^{\circledR}$ (Stryker). In: Pharmazeutische Zeitung online; Govi-Verlag (2007). Im Internet: http://www.pharmazeutischezeitung.de/index.php?id=3408; Stand: 25.03.2013

30 Friedlaender GE, Perry CR, Cole JD et al. Osteogenic protein-1 (bone morphogenetic protein-7) in the treatment of tibial nonunions: a prospective, randomized clinical trial comparing rhOP-1 with fresh bone autograft. J Bone Joint Surg Am 2001; 83 (Suppl. 1): S151-S158

31 McKee MD, Schemitsch EH, Waddell JP et al. The effect of human recombinant bone morphogenic protein (rhBMP-7) on the healing of open tibial shaft fractures: results of a multi-center, prospective, randomized clinical trial. In: Proceedings of the 18th Annual Meeting of the Orthopaedic Trauma Association, Oct 11-13, Toronto, Ontario, Canada; 2002: 157-158
32 Jones AL, Buchholz RW, Bosse MJ et al. Recombinant human BMP-2 and allograft compared with autogenous bone graft for reconstruction of diaphyseal tibial fractures with cortical defects. A randomized, controlled trial. J Bone Joint Surg Am 2006; 88: 1431-1441

33 Calori GM, D'Avino M, Tagliabue L et al. An ongoing research for evaluation of treatment with BMPs or AGFs in long bone non-union: protocol description and preliminary results. Injury 2006; 37: 43-50

34 Ekrol I, Hajducka C, Court-Brown C et al. A comparison of RhBMP-7 (OP-1) and autogenous graft for metaphyseal defects after osteotomy of the distal radius. Injury 2008; 39: 73-82

35 Leach J, Bittar RG. BMP-7 (OP-1) safety in anterior cervical fusion surgery. J Clin Neurosci 2009; 16: 1417-1420

36 Shimer AL, Oncer FC, Vaccaro AR. Spinal reconstruction and bone morphogenetic proteins: open questions. Injury 2009; 40: 32-38

37 Ehnert S, Zhao J, Pscherer $S$ et al. Transforming growth factor beta1 inhibits bone morphogenic protein (BMP)-2 and BMP-7 signaling via upregulation of Ski-related novel protein $\mathrm{N}(\mathrm{SnoN})$ : possible mechanism for the failure of BMP therapy? BMC Med 2012; 10: 101

38 Garrison KR, Shemilt I, Donell S et al. Bone morphogenetic protein (BMP) for fracture healing in adults. Cochrane Database Syst Rev 2010; 6: CD006950

39 Garrison KR, Donell S, Ryder J et al. Clinical effectiveness and cost-effectiveness of bone morphogenetic proteins in the non-healing of fractures and spinal fusion: a systematic review. Health Technol Assess 2007; 30: 1-150

40 Alt V, Heissel A. Economic considerations for the use of recombinant human bone morphogenetic protein-2 in open tibial fractures in Europe: the German model. Curr Med Res Opin 2006; 22 (Suppl.1): S19-S22

41 Luo J, Tang M, Huang J et al. TGFbeta/BMP type I receptors ALK1 and ALK2 are essential for BMP9-induced osteogenic signaling in mesenchymal stem cells. J Biol Chem 2010; 285: 29588-29598

42 Piek E, Heldin C-H, Ten Dijke P. Specificity, diversity, and regulation in TGF- $\beta$ superfamily signaling. FASEB J 1999; 13: 2105-2124

$43 \mathrm{Wu} N$, Zhao Y, Yin Yet al. Identification and analysis of type II TGF-beta receptors in BMP-9-induced osteogenic differentiation of C3H10T1/2 mesenchymal stem cells. Acta Biochim Biophys Sin (Shanghai) 2010; 42: 699-708

44 Kloos DU, Choi C, Wingender E. The TGF- $\beta$-Smad network: introducing bioinformatic tools. Trends Genet 2002; 18: 96-103

45 Miyazawa K, Shinozaki M, Hara T et al. Two major Smad pathways in TGF- $\beta$ superfamily signalling. Genes Cells 2002; 7: 1191-1204

46 Lai CF, Cheng SL. Signal transductions induced by bone morphogenetic protein-2 and transforming growth factor-beta in normal human osteoblastic cells. J Biol Chem 2002; 277: 15514-15522

47 Ross S, Hill CS. How the Smads regulate transcription. Int J Biochem Cell Biol 2008; 40: 383-408

48 Derynck R, Zhang YE. Smad-dependent and Smad-independent pathways in TGF- $\beta$ family signalling. Nature 2003; 425: 577-584

49 Moustakas A. Smad Signalling network. J Cell Sci 2002; 115: 33553356

50 Tang N, Song WX, Luo J et al. BMP-9-induced osteogenic differentiation of mesenchymal progenitors requires functional canonical Wnt/betacatenin signalling. J Cell Mol Med 2009; 13: 2448-2464

51 Javelaud D, Mauviel A. Crosstalk mechanisms between the mitogen-activated protein kinase pathways and Smad signaling downstream of TGF-beta: implications for carcinogenesis. Oncogene 2005; 24: 57425750

52 Zhao Y, Song T, Wang Wet al. P38 and ERK1/2 MAPKs act in opposition to regulate BMP9-induced osteogenic differentiation of mesenchymal progenitor cells. PLoS One 2012; 7: e43383

53 Nguyen J, Tang SY, Nguyen D et al. Load regulates bone formation and Sclerostin expression through a TGFbeta-dependent mechanism. PLoS One 2013; 8: e53813

54 McMahon MS. Bone morphogenic protein 3 signaling in the regulation of osteogenesis. Orthopedics 2012; 35: 920

55 Cook SD. Preclinical and clinical evaluation of osteogenic Protein 1 (BMP-7) in bony sites. Orthopedics 1999; 22: 669-671

56 Geesink RG, Hoefnagels NH, Bulstra SK. Osteogenic activity of OP 1 bone morphogenetic protein (BMP 7) in a human fibular defect. J Bone Joint Surg Br 1999; 81: 710-718

57 Chen G, Yang JZ, Xu HM et al. The application of NNB/BMP complex in the treatment of ununited tibia fracture. Orthopedic Journal of China 2000; 7: 758-761 
58 Govender S, Csimma C, Genant HK et al. Recombinant human bone morphogenetic protein 2 for treatment of open tibial fractures: a prospective, controlled, randomized study of four hundred and fifty patients. J Bone Joint Surg Am 2002; 48: 2123-2134

59 Maniscalco P, Gambera D, Bertone $C$ et al. Healing of fresh tibial fractures with OP1. A preliminary report. Acta Biomed 2002; 73: 27-33
60 Murphy C. Endo-fin-ally a SARA for BMP receptors. J Cell Sci 2007; 120 : 1153-1155

61 Bahamonde ME, Lyons KM. BMP3: to be or not to be a BMP. J Bone Joint Surg Am 2001; 83: 56-62 